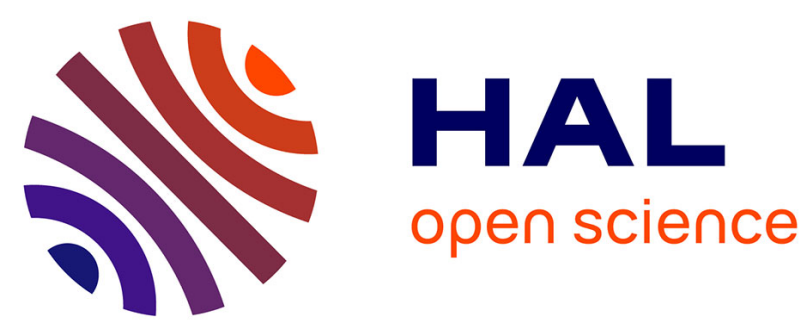

\title{
Cluster observations of broadband ULF waves near the dayside polar cap boundary: Two detailed multi-instrument event studies
}

H. Matsui, P.A. Puhl-Quinn, R.B. Torbert, W. Baumjohann, C. J. Farrugia, C.G. Mouikis, E.A. Lucek, Pierrette Décréau, G. Paschmann

\section{To cite this version:}

H. Matsui, P.A. Puhl-Quinn, R.B. Torbert, W. Baumjohann, C. J. Farrugia, et al.. Cluster observations of broadband ULF waves near the dayside polar cap boundary: Two detailed multiinstrument event studies. Journal of Geophysical Research Space Physics, 2007, 112 (A7), pp.A07218. 10.1029/2007JA012251 . insu-02872192

\section{HAL Id: insu-02872192 \\ https://hal-insu.archives-ouvertes.fr/insu-02872192}

Submitted on 17 Jun 2020

HAL is a multi-disciplinary open access archive for the deposit and dissemination of scientific research documents, whether they are published or not. The documents may come from teaching and research institutions in France or abroad, or from public or private research centers.
L'archive ouverte pluridisciplinaire HAL, est destinée au dépôt et à la diffusion de documents scientifiques de niveau recherche, publiés ou non, émanant des établissements d'enseignement et de recherche français ou étrangers, des laboratoires publics ou privés. 


\title{
Cluster observations of broadband ULF waves near the dayside polar cap boundary: Two detailed multi-instrument event studies
}

\author{
H. Matsui, ${ }^{1}$ P. A. Puhl-Quinn, ${ }^{1}$ R. B. Torbert, ${ }^{1}$ W. Baumjohann, ${ }^{2}$ C. J. Farrugia,${ }^{1}$ \\ C. G. Mouikis, ${ }^{1}$ E. A. Lucek, ${ }^{3}$ P. M. E. Décréau, ${ }^{4}$ and G. Paschmann ${ }^{5}$ \\ Received 3 January 2007; revised 7 May 2007; accepted 18 May 2007; published 26 July 2007.
}

[1] We study two examples of broadband ultra-low frequency (ULF) waves observed near the dayside polar cap boundary using data from multiple instruments on Cluster. These waves lie in a frequency range of $\sim 1-100 \mathrm{mHz}$ with variable amplitudes. We have focused on their characteristics around the lowest frequency end. In the first event, the magnetic variations consist of oscillations mainly transverse to the background field. These waves are inferred to be the shear Alfvén waves whose phase velocity is approximately perpendicular to the magnetic field. A possible source is located in reconnection, probably of transient variety. In the second event, the magnetic variations include compressional components. These waves are suggested to be fast mode waves. It is possible that the waves are generated by the pressure variations in the solar wind impinging on the magnetosphere. The estimated wave modes and generation mechanisms of these two events are different, even though the waves have broadband spectra at similar spacecraft locations. The results from our observations may be useful when comparing with theories in realistic situations.

Citation: Matsui, H., P. A. Puhl-Quinn, R. B. Torbert, W. Baumjohann, C. J. Farrugia, C. G. Mouikis, E. A. Lucek, P. M. E. Décréau, and G. Paschmann (2007), Cluster observations of broadband ULF waves near the dayside polar cap boundary: Two detailed multiinstrument event studies, J. Geophys. Res., 112, A07218, doi:10.1029/2007JA012251.

\section{Introduction}

[2] One category of waves in the magnetosphere is broadband waves, where the power is not restricted to a single frequency. A possible scenario is that waves with multiple frequency components overlap. Another is that the waveforms are variable in time and/or are sporadic so that the original sinusoidal waveform gets distorted. Whereas narrowband ultra-low frequency (ULF) waves in the Pc 1-5 frequency ranges $(2 \mathrm{mHz}-5 \mathrm{~Hz})$ have been widely studied [e.g., Kivelson, 1995, and references therein], broadband waves in the same frequency ranges have received less attention. And yet, in their statistical study of ULF waves in the equatorial magnetosphere at $L=5-9$ by AMPTE/ CCE, Anderson et al. [1990] reported that $30-40 \%$ of all spectra at frequency $<80 \mathrm{mHz}$ were classified as broadband. Engebretson et al. [1995] analyzed one category of broadband ULF waves, Pi 1-2 pulsations, on the dayside. They used ground magnetometer data from stations situated

\footnotetext{
${ }^{1}$ Space Science Center, University of New Hampshire, Durham, New Hampshire, USA.

${ }^{2}$ Space Research Institute, Austrian Academy of Sciences, Graz, Austria.

${ }^{3}$ Imperial College, London, UK

${ }^{4}$ LPCE, CNRS, University of Orléans, Orléans, France.

${ }^{5}$ Max-Planck-Institut für extraterrestrische Physik, Garching, Germany.
}

Copyright 2007 by the American Geophysical Union. 0148-0227/07/2007JA012251 at magnetic latitudes of $75-79^{\circ}$ and in a magnetic local time (MLT) band of $\sim 6$ hours. It was reported that this type of broadband wave activity increases with the solar wind speed. One possible source of these waves was suggested to be the Kelvin-Helmholtz instability, related to flow shears between the magnetosheath and the magnetosphere. Another report that the flow shears could cause growth of kinetic Alfvén waves was given by $\mathrm{Pu}$ and Zhou [1986]. Farrugia et al. [1989] reported ULF geomagnetic perturbations at ground stations at $67-75^{\circ}$ magnetic latitudes driven by magnetopause oscillations caused by solar wind pressure variations. Based on this observation, Southwood and Kivelson [1990] developed a model in which magnetopause motions launch fast mode waves into the magnetosphere. In this model, the fast mode waves couple to Alfvén waves in an inhomogeneous background. Chaston et al. [2003] reported smallscale Alfvén waves recorded by FAST at the cusp and polar cap boundary of the premidnight auroral oval. One possible generation mechanism of these Alfvén waves was suggested to be magnetic reconnection [Chaston et al., 2003]. Subsequently, Chaston et al. [2005] reported kinetic Alfvén waves with a broad frequency band from data acquired by Cluster at $\sim 5 R_{E}$ altitude and combining them with FAST data. It was proposed that the waves originally generated as surface waves driven by the Kelvin-Helmholtz instability are mode converted. Bosqued et al. [2005] reported small-scale fieldaligned currents causing magnetic perturbations perpendicular to the ambient magnetic field by using data from 
Cluster at an altitude of $\sim 5 R_{E}$. These structures were suggested to result from magnetic reconnection at the magnetopause.

[3] As can be seen from the above brief overview, the mode and source of the broadband ULF waves could be various. In this study, we select two events of broadband waves observed with the wave power in the $\sim 1$ - to $100-\mathrm{mHz}$ frequency range above the background level by Cluster. We especially focus on the lowest frequency end of these waves to highlight their difference, even though the spacecraft locations are similar in the two events. This type of comparative study would lead to a deeper understanding of the broadband waves. It should be noted that there is an effort for a comprehensive study on the waves in a similar frequency range [Puhl-Quinn et al., 2004]. Cluster offers the following advantages which allow for a sensitive analysis of the waves: (1) Magnetic field data from multiple spacecraft are available, which makes it possible to determine the phase velocity from the magnetic field data alone [Eriksson, 1998; Soucek et al., 2004; Bosqued et al., 2005] using a principle described in Born and Wolf [1999]. (2) Electric field data are also available, so that we can determine the amplitude ratio of electric to magnetic fluctuations, the Poynting flux, and the convection velocity of the background plasma. (3) Alfvén velocity can be derived by combining particle and magnetic field data. When we combine the above observations, we can examine the mode of the broadband waves. We shall suggest that the two events consist of different modes, shear Alfvén and fast modes, respectively. A possible identification of the fast mode waves around the dayside polar cap boundary is a new feature of Cluster observations. Based on this analysis, we will try to estimate the source of the waves by referring to the available interplanetary parameters. Finally, concluding remarks are drawn, and some theoretical implications are provided.

\section{Data}

[4] In this study, we use magnetic field data from the Fluxgate Magnetometer (FGM) on Cluster [Balogh et al., 2001] at spin period ( $\sim 4 \mathrm{~s})$ resolution. We also use electric field data from the Electron Drift Instrument (EDI) [Paschmann et al., 2001], the maximum time resolution of which is $\sim 1 \mathrm{~s}$. There are intermittent data gaps because electron beams released from the electron guns do not always return to the detectors. However, the EDI data are relatively immune to plasma-spacecraft interactions that can affect the double-probe data at polar caps [Eriksson et al., 2006]. The Nyquist frequency of the magnetic field data is $125 \mathrm{mHz}$ so that we can investigate waves below this frequency, at which broadband ULF waves are known to exist. The data are presented either in solar magnetospheric (SM) coordinates or in magnetic field-aligned coordinates, as appropriate. In the latter coordinates, radial (inward positive), azimuthal (eastward positive), and parallel components will be shown. Finally, data from the Cluster Ion Spectrometry (CIS) [Rème et al., 2001] and the Waves of High frequency and Sounder for Probing of Electron density by Relaxation experiment (WHISPER) [Décréau et al., 2001] are used in order to estimate the Alfvén velocity and the ion gyroradius. We take into account contribution from oxygen ions when calculating the Alfvén velocity with the following equation:

$$
V_{A}=\frac{B}{\mu_{0} \sqrt{m_{p}\left(n_{e}-n_{o}\right)+m_{o} n_{o}}}
$$

where $B$ is the magnitude of the magnetic field, $\mu_{0}$ is the magnetic permeability, $m_{\mathrm{p}}$ is the proton mass, $n_{\mathrm{e}}$ is the electron density, $n_{\mathrm{o}}$ is the oxygen ion density, and $m_{\mathrm{o}}$ is the oxygen mass. Here we assume all $\mathrm{O}^{+}$ions are within the energy range of the detectors $(40 \mathrm{eV}-40 \mathrm{keV})$.

\section{Observations}

[5] Figure 1 shows frequency-time spectra of magnetic variations and time series of background magnetic and electric fields for 1600-2000 UT, 26 March 2001, acquired by spacecraft 1 . Frequency-time spectra for magnetic fields are shown for radial $\left(B_{\perp} r\right)$, azimuthal $\left(B_{\perp a}\right)$, and fieldaligned $\left(B_{\|}\right)$components in the top three panels. The bottom two panels show magnetic and electric fields for magnitudes (black line), elevation (red line), and azimuth (green line). The magnetic field strength shown in the fourth panel decreases smoothly as the spacecraft moves from the polar cap toward its boundary in the dawnside northern hemisphere. The magnetopause crossing occurs at $\sim 1920$ UT, after which the magnetic fluctuations increase. The electric field shows large oscillations compared to the magnetic field on top of the background component. The direction of convection is dawnward before $\sim 1720$ UT and antisunward after this time, indicating that the spacecraft is located inside the dawn cell. The magnetic field spectra show activity in the whole frequency range of approximately two decades, indicating that the waves are broadband. The transverse components tend to have larger amplitude than the compressional components. As the spacecraft approaches the magnetopause, the amplitudes of both transverse and compressional components increase. In the subsequent analysis, we concentrate on the 30 -minute period at 1800-1830 UT, indicated by vertical dotted lines. This we do for the following reasons. (1) There is moderate wave power in both magnetic and electric fields. (2) The magnetic field from multiple spacecraft tends to have similar oscillations with a relatively stable time lag so that the phase velocity can be determined well during this period.

[6] Figure 2 shows time series plots of the magnetic field and electric field oscillations for the time interval from 1800 to $1830 \mathrm{UT}$. We have subtracted 300 -s running averages from the original data. The top three panels show magnetic fields for radial $\left(B_{\perp} r\right)$, azimuthal $\left(B_{\perp a}\right)$, and field-aligned $\left(B_{\|}\right)$components. The next two panels show the radial $\left(E_{\perp r}\right)$ and azimuthal $\left(E_{\perp a}\right)$ components of electric fields. The bottom panel shows the relative configurations between the spacecraft projected onto the $X-Y$ and $Y-Z$ planes in SM coordinates. The data from the four spacecraft are plotted with the following color code: spacecraft 1 (black), 2 (red), 3 (green), and 4 (blue). When the magnetic field-aligned coordinates are defined in the multiple spacecraft analysis, we refer to geometrical mean of the magnetic fields from the four spacecraft. The spacecraft are located over the northern polar cap boundary at $\sim 0850$ MLT and at radial distance of 


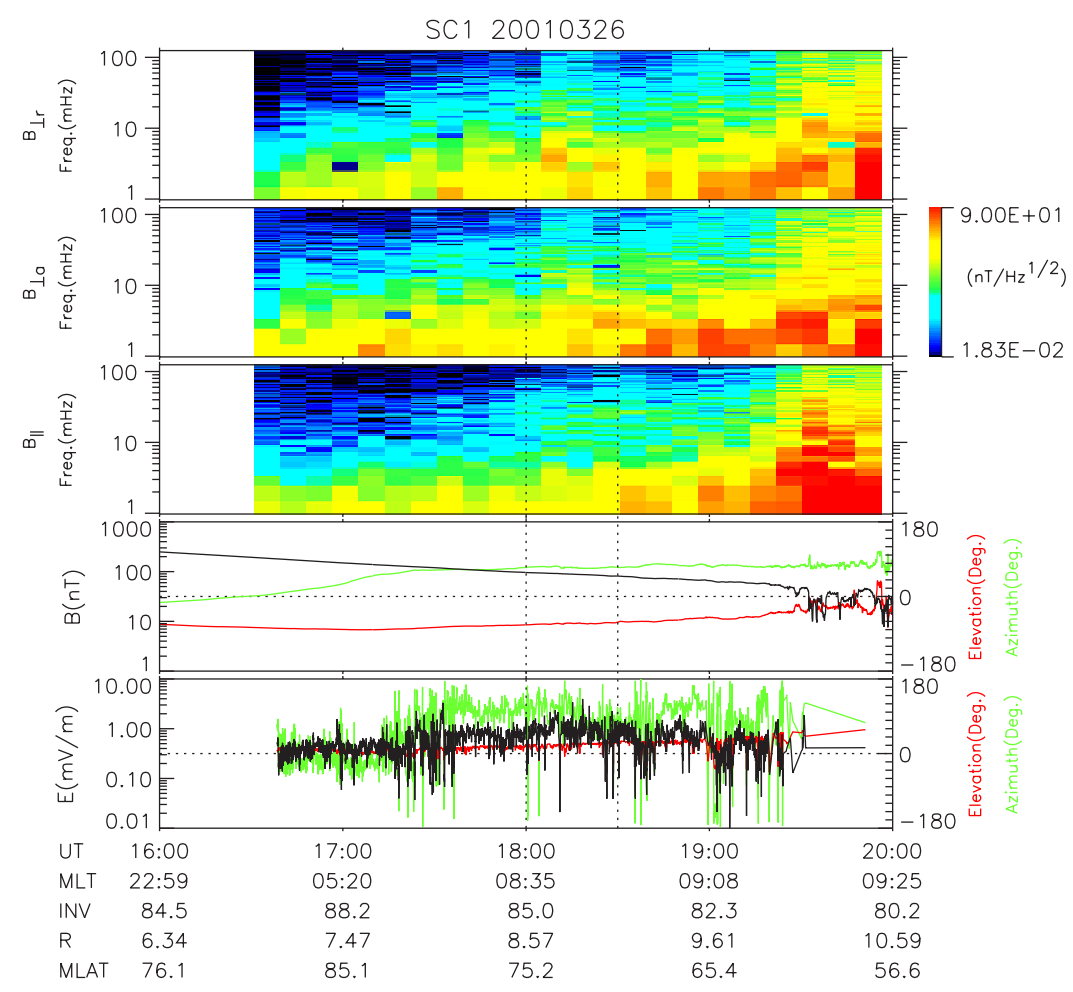

Figure 1. Magnetic field spectra in field-aligned coordinates and time series of magnetic and electric fields in SM coordinates for 1600-2000 UT, 26 March 2001. The interval between the two dotted lines are analyzed in detail.

$\sim 9 R_{E}$. The interplanetary magnetic field at ACE [Smith et al., 1998] shows a slightly negative, fluctuating $B_{Z}$, pointing duskward and tilted antisunward. The solar wind velocity from Solar Wind Electron Proton Alpha Monitor (SWEPAM) [McComas et al., 1998] records a low speed $(320 \mathrm{~km} / \mathrm{s})$. Number density is not available from ACE during this period. The broadband nature of the waves is consistent with the time series magnetic fields in Figure 2 in which the frequency and the amplitude of the waves are variable in time, although the variation of typical period of $\sim 5$ minutes may be discerned. One may note that the amplitude of perpendicular components is larger than that of parallel component. There is a clear shift from spacecraft to spacecraft in the perpendicular components. The magnetic oscillations are observed first at spacecraft 3 and then at spacecraft 1,2, and 4 in this order. When we consult the relative configurations between the spacecraft shown in the bottom panel, it transpires that the perturbation is propagating approximately in the $-X$ direction (antisunward). The propagation velocity is estimated to be in the order of $\sim 10 \mathrm{~km} / \mathrm{s}$ by comparing time shift of magnetic variations between spacecraft 3 and 4 ( $\sim 90 \mathrm{~s})$, for example, and their separation $(\sim 700 \mathrm{~km})$. In the next paragraph, we quantify this value more carefully.

[7] When we refer to either radial or azimuthal component of the magnetic fields, it is possible to determine the phase velocity $\omega / k$ by the following matrix relation, in which we assume a single plane wavefront:

$$
\Delta \phi / \omega=\Delta \boldsymbol{r} \cdot \boldsymbol{k} / \omega
$$

where $\Delta \phi$ is the phase difference of the selected magnetic component between all six pairs of spacecraft $\left(\Delta \phi_{12}, \Delta \phi_{13}\right.$, $\left.\Delta \phi_{14}, \Delta \phi_{23}, \Delta \phi_{24}, \Delta \phi_{34}\right)$. The suffices identify the spacecraft with reference to which the difference is derived. These values are determined by fast Fourier transform for each frequency component. Quantity $\omega$ is the angular frequency. $\Delta \boldsymbol{r}$ is a $6 \times 3$ matrix of the six separation vectors $\left(\Delta \boldsymbol{r}_{12}, \Delta \boldsymbol{r}_{13}, \Delta \boldsymbol{r}_{14}, \Delta \boldsymbol{r}_{23}, \Delta \boldsymbol{r}_{24}, \Delta \boldsymbol{r}_{34}\right)$. Quantity $\boldsymbol{k}$ is the wave number vector. Quantity $\omega / k$ is estimated by minimizing the variance $|\Delta / \omega-\Delta \mathrm{r} \cdot \boldsymbol{k} / \omega|^{2}$, for each of radial and azimuthal components of magnetic fields. We average this quantity when the coherence exceeds 0.8 for the Fourier components at frequencies between 3.9 and $15.6 \mathrm{mHz}$. It should be noted that the coherence is mostly small in the high-frequency ranges above $15.6 \mathrm{mHz}$. The result obtained is shown in Figure 3. Phase velocities estimated from the two magnetic components are shown by different symbols. The directions of the phase velocities corresponding to those of smallest phase speeds are approximately parallel to the $-X$ direction, as anticipated from Figure 2, with a mean value of $(-18.9,5.2,-0.8) \mathrm{km} / \mathrm{s}$ in SM coordinates. These values are within the same order of the convection velocity obtained from the EDI instrument on spacecraft 1 and shown by a solid line in Figure 3. (A mean value for convection is $-10.1,2.8,-0.6 \mathrm{~km} / \mathrm{s}$.) The phase velocity in the plasma frame is calculated as $-9.5,2.8,-0.5 \mathrm{~km} / \mathrm{s}$. The average propagation angle relative to the magnetic field is $92 \pm 10^{\circ}$. The frequency in the plasma frame is lower (59\%) than the frequency in the spacecraft frame because of the Doppler shift. 


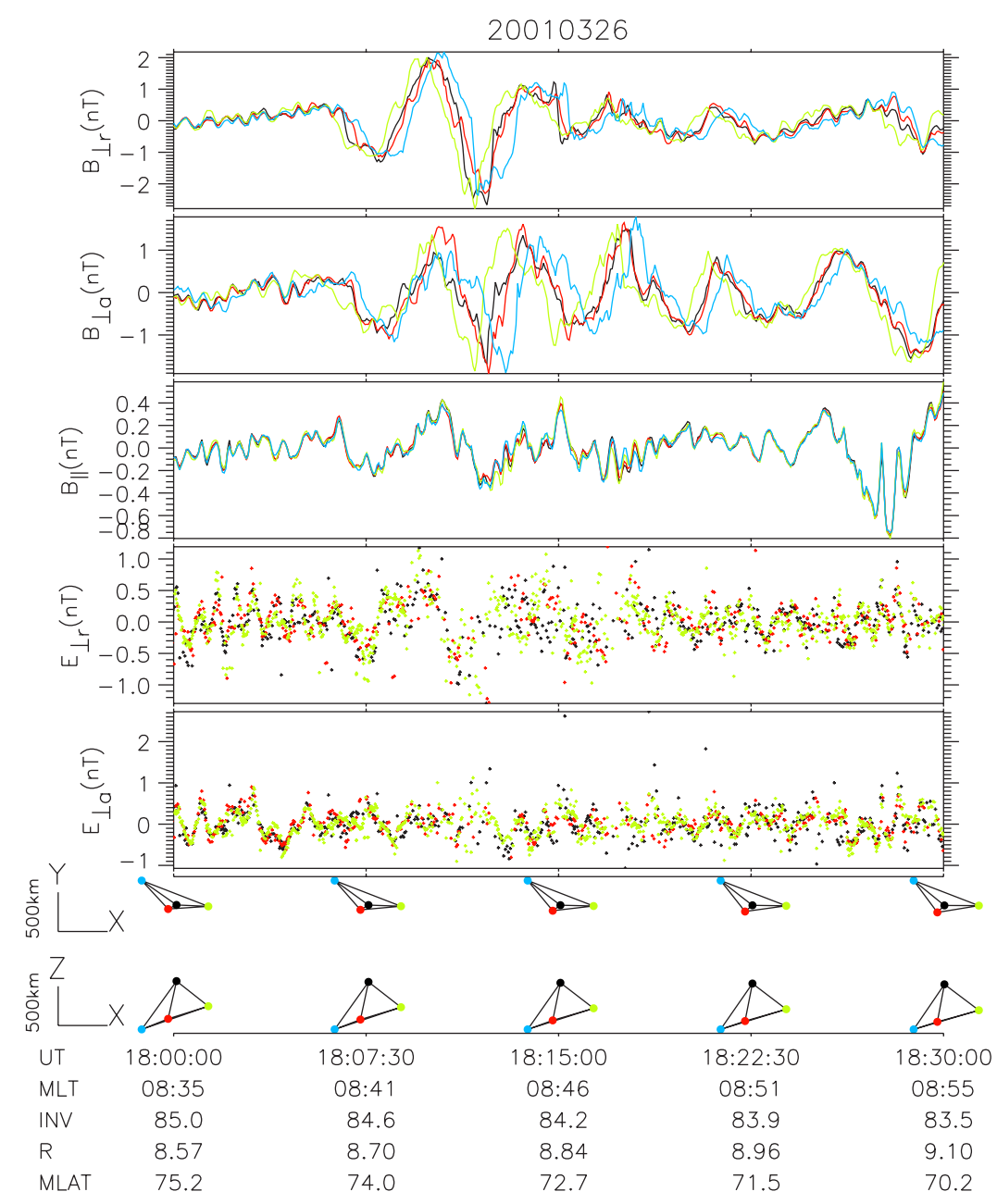

Figure 2. Time series of magnetic field and electric field variations between 1800 and 1830 UT on 26 March 2001. The data from multiple spacecraft are shown in magnetic field-aligned coordinates with the following colors: spacecraft 1 (black), 2 (red), 3 (green), and 4 (blue). The configuration of the four spacecraft is also shown.

[8] In the bottom two panels of Figure 2, two components of electric fields perpendicular to the magnetic fields are plotted for spacecraft 1,2 , and 3 . (The electric field data from spacecraft 4 are not available.) Although there are some data gaps, there is an oscillatory variation similar to that in the magnetic fields especially in the radial component. Slight signatures of time lags between spacecraft are seen at $\sim 1813$ for the radial component and at $\sim 1807$ for the azimuthal component.

[9] Combining the magnetic field and electric field data, we can estimate the amplitude ratio of electric to magnetic fluctuations, $E / B$, and the Poynting flux, $\boldsymbol{S}=E \times B / \mu_{0}$ (Figure 4). Here we chose a band-pass filter between periods of 60 and $300 \mathrm{~s}$, based on calculation of running averages. We limit our interest on wave periods with coherent oscillation between spacecraft. The top panel shows the $E / B$ ratio from three spacecraft (spacecraft 1, 2, and 3 ) and the Alfvén velocity from spacecraft 1 (orange line). The next three panels show radial $\left(\boldsymbol{S}_{\perp} r\right)$, azimuthal $\left(\boldsymbol{S}_{\perp a}\right)$, and field-aligned $\left(\boldsymbol{S}_{\|}\right)$components of the Poynting flux, respectively. The $E / B$ ratio in the top panel has an average value of $540 \mathrm{~km} / \mathrm{s}$ for spacecraft 1, which is much larger than the phase velocity estimated from the magnetometer data. The Alfvén velocity from the same spacecraft shown by an orange line gives an average value of $1270 \mathrm{~km} / \mathrm{s}$, which is larger than the $E / B$ ratio. The bottom three panels show that the Poynting flux is mostly field aligned. The ionospheric reflection of these waves is supported from (1) the discrepancy between the $E / B$ ratio and the Alfvén velocity and (2) the oscillation of the direction of the Poynting flux. Detailed discussion is given in the next section.

[10] We have concentrated on the analysis of these broadband waves for 30 minutes between 1800 and 1830 UT. In addition, the same type of waves are observed between 1740 and 1920 UT. These waves have a phase velocity with the same order as the convection velocity. The $E / B$ ratio tends to be less than the Alfvén velocity, and the Poynting flux is mainly field aligned. However, we should note that the waves with Poynting flux in the perpendicular direction exist especially around the magnetopause. It is possible that other types of waves might coexist during this period.

[11] The second event is shown for 2000-2400 UT, 4 March 2001 (Figure 5), in the afternoon MLT sector in the southern hemisphere. The format of this figure is the same as Figure 1. The spacecraft 1 was first located inside the 


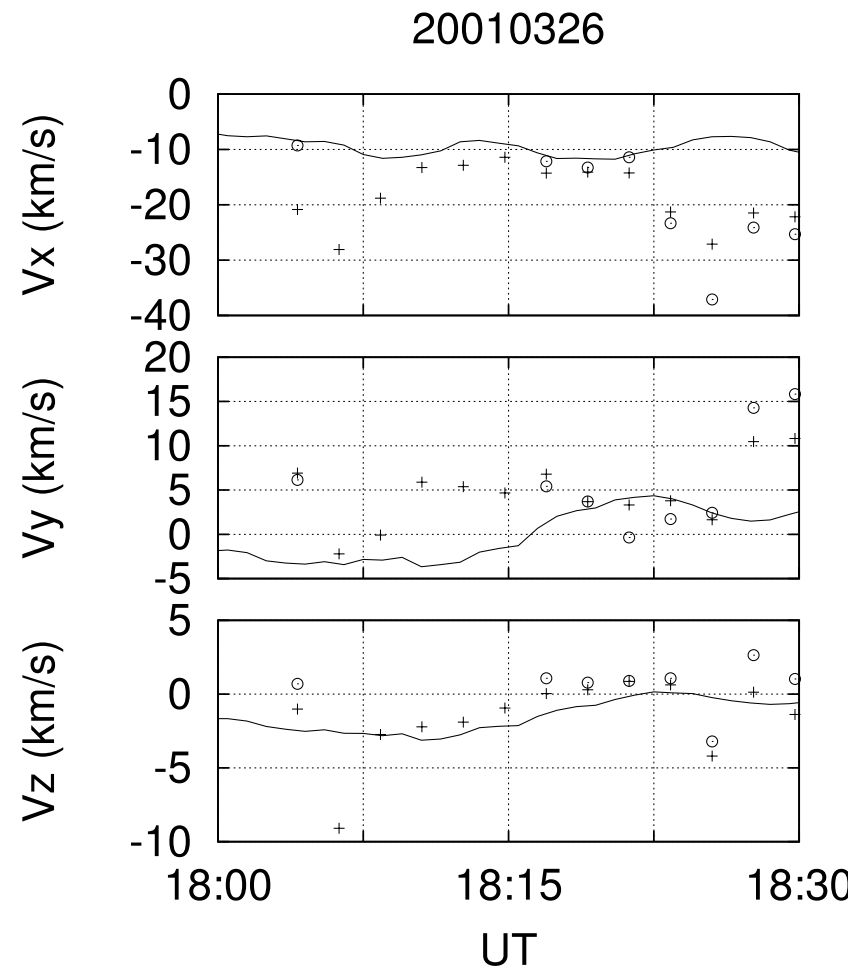

Figure 3. Phase velocity of the waves derived from radial component (plusses) and azimuthal component (circles) of magnetic field variations between 1800 and 1830 UT on 26 March 2001. The convection velocity from EDI on spacecraft 1 is shown with solid lines.

magnetosheath, while fluctuating magnetic fields are evident. Then magnetopause is crossed at $\sim 2200$ UT. The magnitude of the magnetic field increases as the spacecraft enters the polar cap region while descending in radial distance. The electric field is fluctuating and in the duskward direction, indicating that the convection is antisunward. At $\sim 1300 \mathrm{MLT}$, this is consistent with the typical two-cell convection. The magnetic wave component exists in a broad frequency band with a strong power in the magnetosheath. The power diminishes as the spacecraft moves deeper into the magnetosphere. The compressional components are as strong as the transverse components. In this study, we chose the 30-minute interval at 22002230 UT in which there are enough EDI data, and the magnetic variations at the multiple spacecraft are similar between each other.

[12] Filtered time series of magnetic and electric fields are shown in Figure 6 for the selected interval. The format of this figure is the same as Figure 2. The spacecraft are in the southern polar cap boundary at $\sim 1330$ MLT at a radial distance of $\sim 8 R_{E}$. The interplanetary data from ACE show strongly southward IMF $B_{Z}(<-5 \mathrm{nT})$. The IMF $B_{Y}$ component is mostly positive. The solar wind speed is $\sim 440 \mathrm{~km} / \mathrm{s}$. Dynamic pressure varies between 2 and $5 \mathrm{nPa}$. Properties of the waves in the figure are summarized as follows. The magnetic variations are again of variable frequency and amplitude, which is consistent with the broadband spectra in Figure 5. However, unlike the previous example, the magnetic variations are observed in both perpendicular and parallel components and at a comparable amplitude. The magnetic variations are similar at the four spacecraft. It is hard to determine the phase velocity from the magnetic components because of the following limitation on the method. The noise level of the magnetometer is $0.1 \mathrm{nT}$. If there is a magnetic variation with $1 \mathrm{nT} /$ minute, the uncertainty of the timing is $6 \mathrm{~s}$. When the spacecraft separation is $\sim 500 \mathrm{~km}$, the upper limit of the phase velocity which can be determined reliably is estimated as $\sim 80 \mathrm{~km} / \mathrm{s}$. As for the electric fields in Figure 6, oscillations are observed in both radial and azimuthal components and are coincident with the

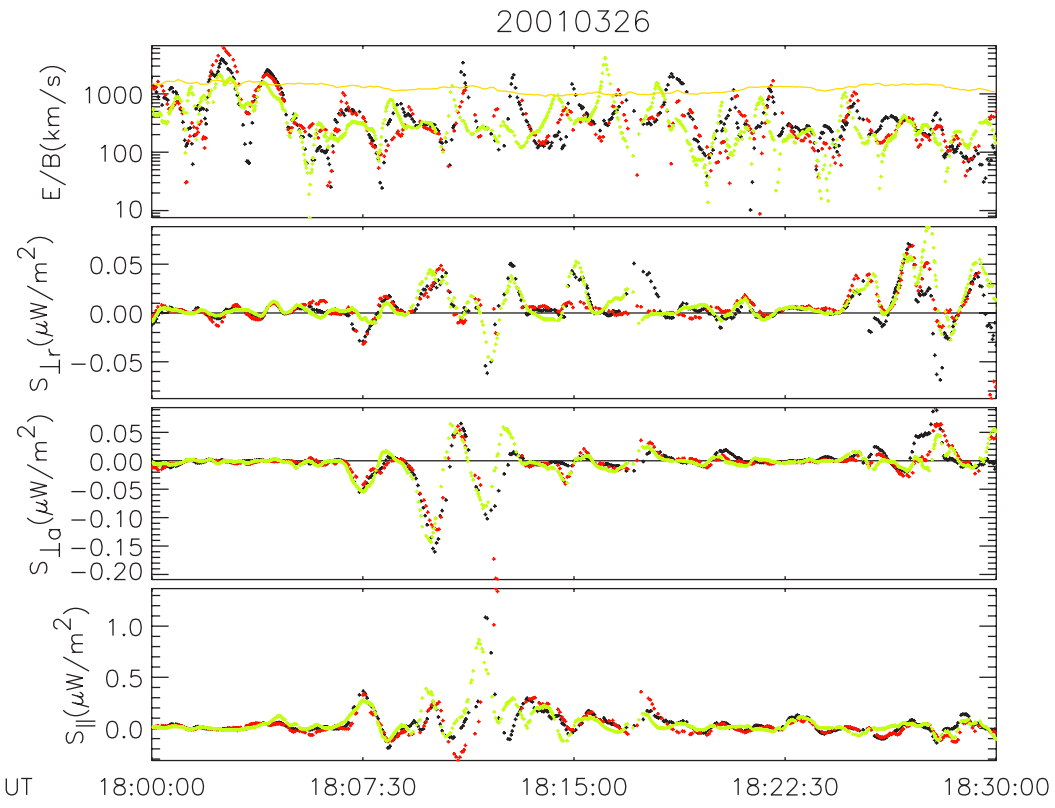

Figure 4. $E / B$ ratio and Poynting flux $\boldsymbol{S}$ between 1800 and 1830 UT on 26 March 2001. The Alfvén velocity from spacecraft 1 is shown with an orange line in the top panel. 


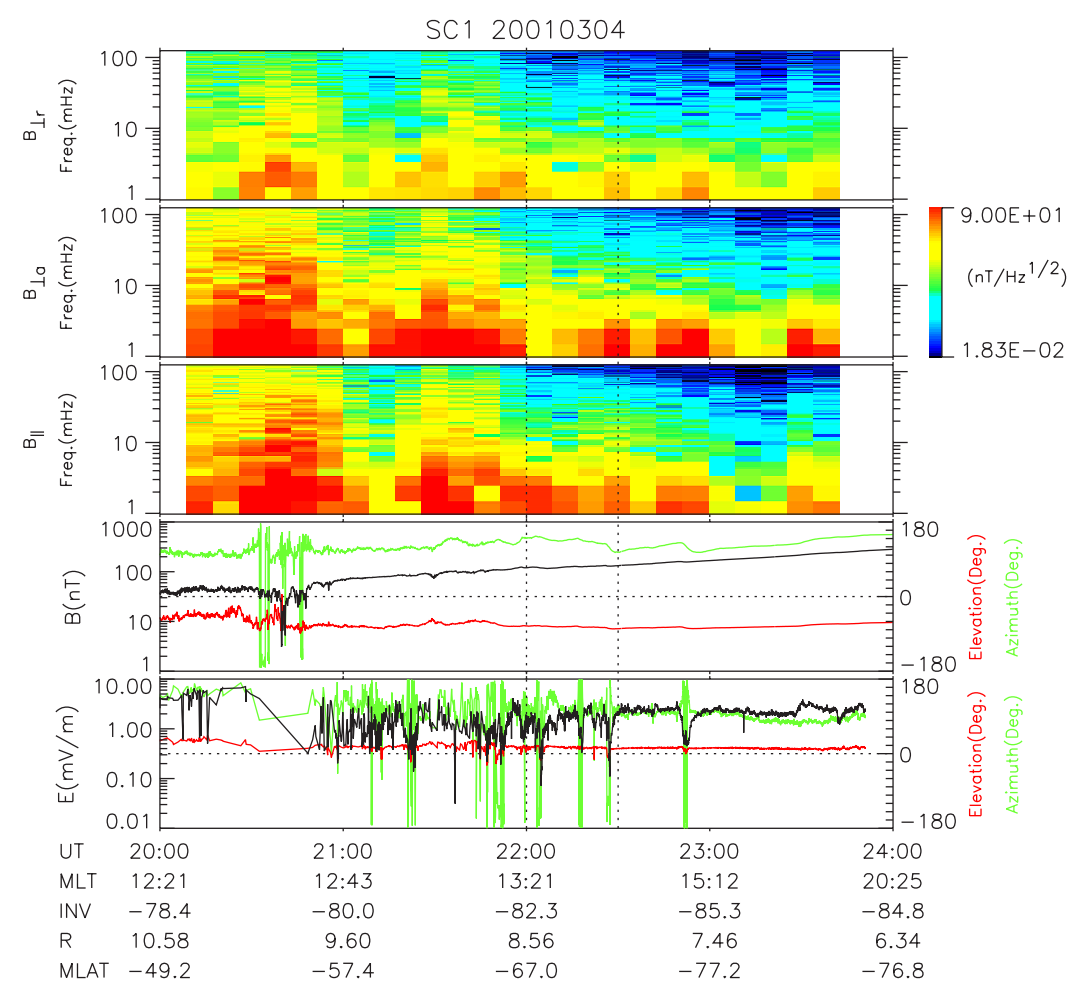

Figure 5. Magnetic field spectra in field-aligned coordinates and time series of magnetic and electric fields in SM coordinates for 1600-2000 UT, 26 March 2001. The interval between the two dotted lines is analyzed in detail.

magnetic oscillations. The oscillations from multiple spacecraft are similar between each other.

[13] The calculated $E / B$ ratio, Alfvén velocity, and Poynting flux are shown in Figure 7. The format of this figure and the periods for band-pass filters are the same as the previous event analysis for comparative purpose. The $E / B$ ratio has an average value of $460 \mathrm{~km} / \mathrm{s}$ for spacecraft 1 , while the Alfvén velocity is calculated as $1030 \mathrm{~km} / \mathrm{s}$. The Poynting flux has a large component in the perpendicular direction, which is also comparable to that in the parallel direction. The direction of the energy propagation is thus mainly inward and eastward (antisunward), although this direction sometimes changes. Since the spacecraft are located at $\sim 1330$ MLT, the wave energy propagates from the direction of the subsolar magnetopause in earlier MLT.

[14] In addition to the interval investigated in detail between 2200 and 2230 UT, we have observed waves with similar characteristics in the longer interval between 2110 and $2300 \mathrm{UT}$. The $E / B$ ratio is less than the Alfvén velocity. Perpendicular Poynting flux can be identified. The magnetic field observation from multiple spacecraft does not show a phase lag. However, the other types of waves, the waves with a phase lag, are sometimes observed. This does not contradict the IMF conditions, where IMF $B_{Z}$ was strongly negative, in which case Alfvén waves might be excited as discussed below.

\section{Discussion}

[15] We have reported two events of broadband ULF waves observed by Cluster near the dayside polar cap boundary. Characteristics of these waves are examined in detail at the lowest frequency end. In this section, we discuss possible modes and generation mechanisms of these waves for each event.

\subsection{The Event on 26 March 2001}

[16] From the reported observations, the waves are inferred to be shear Alfvén waves whose phase velocity is approximately orthogonal to the ambient magnetic field. Various characteristics of Alfvén waves are now compared with our observations. (1) The frequency range is much smaller than the ion cyclotron frequency so that the magnetohydrodynamic (MHD) approximation holds. In our case, the ion cyclotron frequency from the magnetic field measurement is $1.3 \mathrm{~Hz}$, while the frequency we deal with is much lower. (2) The magnetic field perturbation is transverse to the ambient magnetic field. This is also what we observed (Figure 2). (3) The phase velocity is equal to the Alfvén velocity when the propagation angle is parallel to the background magnetic field. As the angle between the phase velocity and the magnetic field increases to $90^{\circ}$, the phase velocity decreases to $0 \mathrm{~km} / \mathrm{s}$. Our observation indicates that the phase velocity is much smaller than the Alfvén velocity and is therefore close to the direction perpendicular to the magnetic field. (4) The $E / B$ ratio is equal to the Alfvén velocity. This is not well satisfied in our observations (Figure 4 ) where the $E / B$ ratio is $\sim 43 \%$ of the Alfvén velocity. (5) The magnetic field and electric field perturbations are perpendicular to each other. This does not always hold in our case because the magnetic variation is along the 


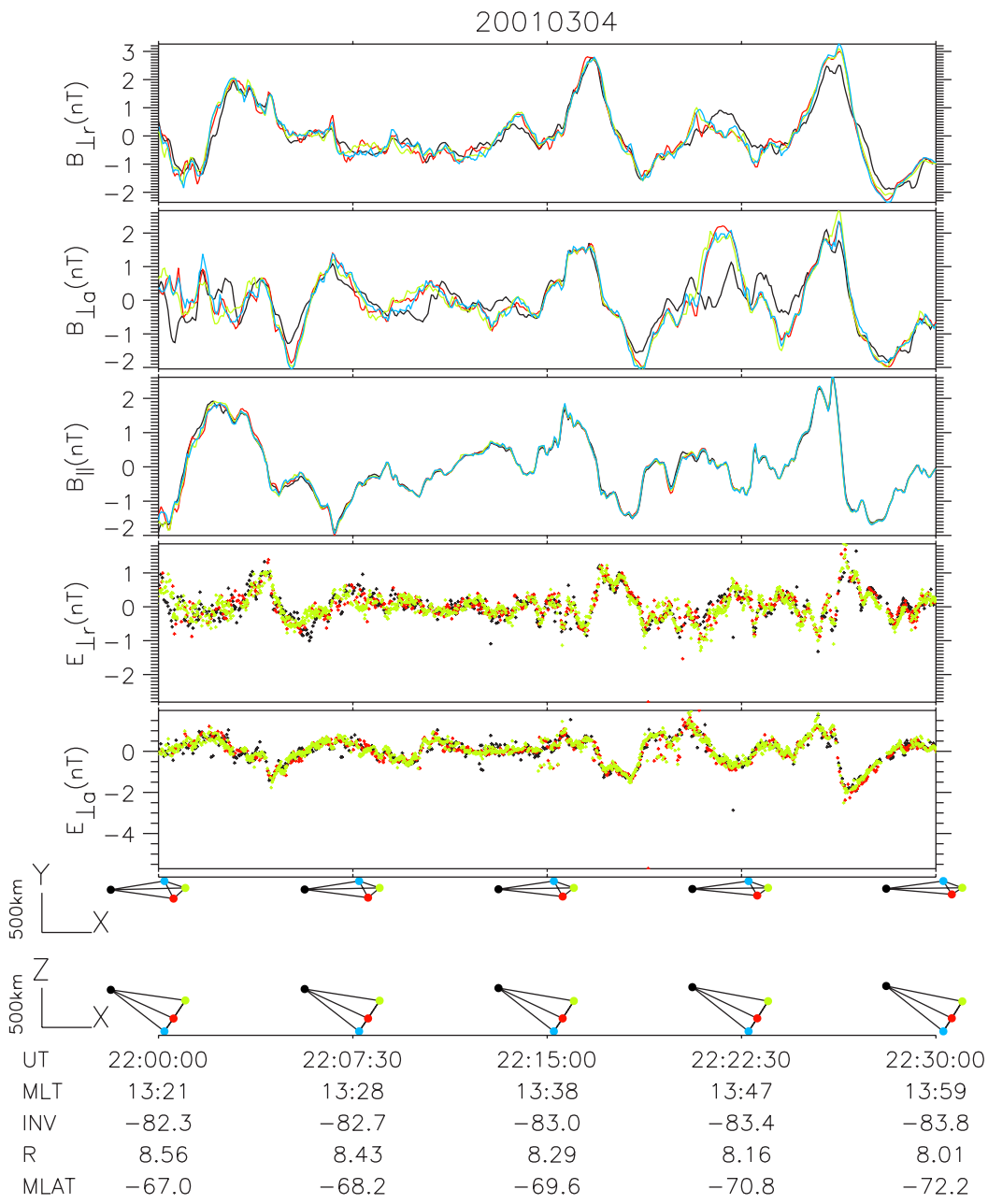

Figure 6. Time series of magnetic field and electric field variations between 2200 and 2230 UT on 4 March 2001. The configuration of the four spacecraft is also shown.

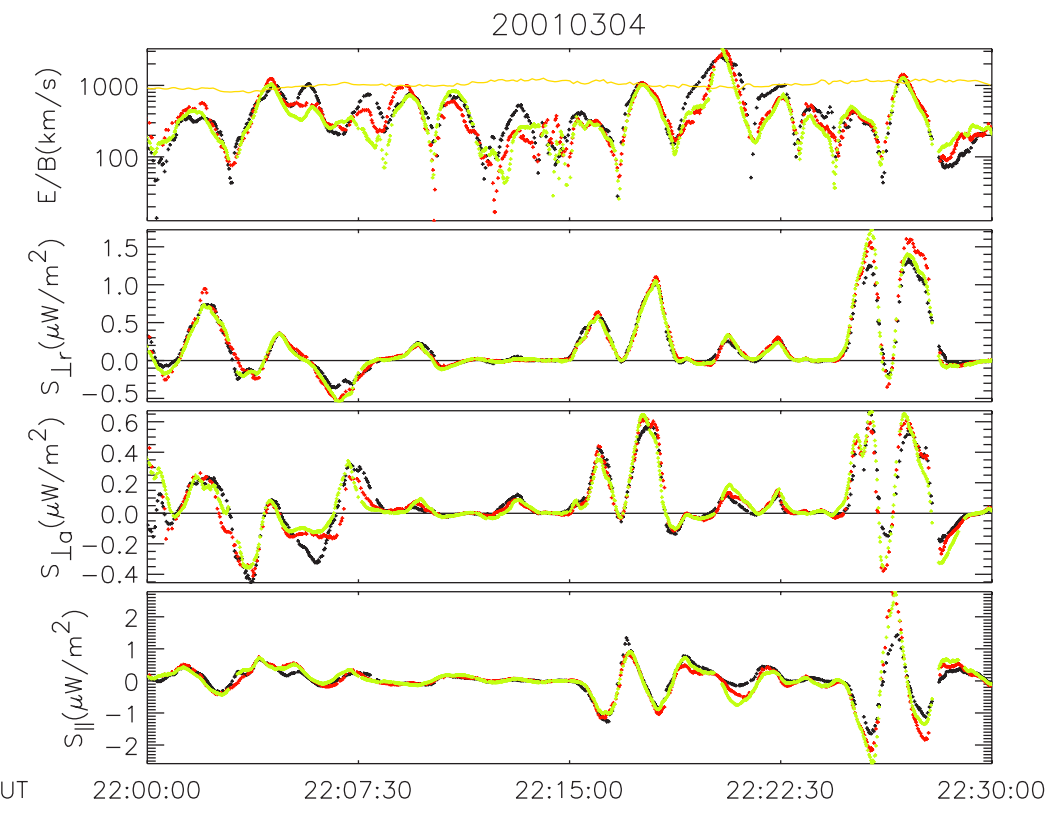

Figure 7. $E / B$ ratio and Poynting flux $\boldsymbol{S}$ between 2200 and 2230 UT on 4 March 2001. The Alfvén velocity from spacecraft 1 is shown with an orange line in the top panel. 
$X$ direction, while the electric variation is along the radial direction (Figure 2).

[17] We now interpret the above comparisons. While points 1-3 support the observation of Alfvén waves, points 4 and 5 do not do so straightforwardly. There are several reasons for this. (1) The waves with energy propagating toward and away from the ionosphere coexist. The $E / B$ ratio can change along the field line, such as happens in cases of field line resonances through ionospheric coupling and reflection [e.g., Lysak, 1991; Chaston et al., 2005]. The E/B ratio would be different from the Alfven velocity in this case. As the waves with multiple directions coexist, the angle between the electric and magnetic field directions can take any value. It is possible that the magnetic and electric variations are not always similar to each other because of the variable amplitudes between waves traveling in opposite directions. (2) There is also the possibility that the Alfvén waves are affected by the ionospheric Pedersen current. Thus Dombeck et al. [2005] estimated the $E / B$ ratio caused by this ionospheric current as $800 / \Sigma_{p} \mathrm{~km} / \mathrm{s}$, where $\Sigma_{p}$ is the Pedersen conductivity in mhos at the ionospheric foot point. There are several contributions to this conductivity, such as the solar illumination and auroral electron precipitation. Here we calculate the contribution from the solar illumination as $\sim 4$ mho by referring to the study of Robinson and Vondrak [1984], which gives a lower limit on the conductivity because of the neglect of the contribution from the electron precipitation. In this case, the $E / B$ ratio caused by the ionospheric current is $200 \mathrm{~km} / \mathrm{s}$ which is smaller than the observed $E / B$ ratio, $540 \mathrm{~km} / \mathrm{s}$. As the $E / B$ ratio lies between the expectation of the ionospheric current closure and the Alfvén velocity, it is possible that the Alfvén waves at the Cluster location are affected by the ionospheric current. (3) If the static current structure exists without any connection to the ionosphere, only the magnetic field variation could appear, which is one way of lowering the $E / B$ ratio. However, this possibility is implausible because a static field-aligned current system is unlikely to be closed by diamagnetic or gradient drift currents, given the very low plasma $\beta$. (4) A further possibility is that the Alfvén waves are modified by kinetic effects due to the finite gyroradius of ions. Our observed phase velocity is approximately in the direction perpendicular to the magnetic field, which is one property of kinetic Alfvén waves. However, this kinetic effect causes the $E / B$ ratio to exceed the Alfvén velocity [e.g., Stasiewicz et al., 2001; Chaston et al., 2005]. The average value of $1 / k$ in our example is $560 \mathrm{~km}$, while that of the proton gyroradius is $7.2 \mathrm{~km}$. If the waves are not damped, the wave mode needs to be supported by the above local conditions to be identified as the kinetic Alfvén waves. We conclude that the wave properties are not modified much by kinetic effects. Kinetic effects might be important at other locations along the magnetic field line, such as the generation region of the waves [e.g., Chaston et al., 2005].

[18] Next we consider generation mechanisms of the shear Alfvén waves. It is possible that the reconnection process is the source of the waves because the Alfvén waves are known to form part of the reconnection layer [e.g., Heyn et al., 1988]. In addition, the spacecraft and the expected location of the reconnection at the magnetopause are located in the same MLT range and hemisphere. For a duskward and slightly southward IMF, the reconnection site is expected to be at the morningside magnetopause in the northern hemisphere [e.g., Gosling et al., 1990]. The magnetic tension from the reconnection site at the magnetopause propagates down toward the ionosphere as Alfvén waves, which carry field-aligned current [Cowley, 2000]. The waves we observed are related to the field-aligned current because the phase velocity is in the direction approximately perpendicular to the magnetic field. The currents related to the waves are expected to be perpendicular to both the directions of the phase velocity and the magnetic perturbation. In the cusp region, a Cluster observation of small-scale field-aligned currents possibly caused by the reconnection has been reported [Bosqued et al., 2005], which is similar to our observation. It should be noted that the kinetic effect might be important for the generation of these waves because of the Hall instability at the reconnection site [Stasiewicz et al., 2001]. Another point to be noted is that the Poynting flux is dominant only during $~ 1810-1813$ UT. Thus the shear Alfvén waves excited by a reconnection process are transient.

[19] These magnetic variations could have different origin from the waves generated by velocity shears at the magnetopause [Engebretson et al., 1995]. The solar wind speed in our case is $\sim 320 \mathrm{~km} / \mathrm{s}$. Thus the flow shear instability is unlikely to be responsible for generating these waves.

\subsection{The Event on 4 March 2001}

[20] Four modes may be considered for these waves. Of these, the Alfvén and slow modes are ruled out because there is Poynting flux in the perpendicular direction. The third mode, the drift mirror mode, has a compressional component. However, this mode is also ruled out because the instability condition [Hasegawa, 1969] is not satisfied. The fast mode, the fourth possibility, agrees more with the observation. Fast mode waves have the following characteristics, which are now compared with our observations. (1) The waves belong to the MHD mode. This condition is satisfied because the wave frequency is much smaller than the ion cyclotron frequency, $1.9 \mathrm{~Hz}$. (2) The magnetic perturbation could include compressional components, as we actually observed (Figure 6). (3) The phase velocity is equal to the Alfvén velocity when the contribution from the ion acoustic velocity is negligible, which is consistent with our result. (The ion acoustic velocity is $41 \mathrm{~km} / \mathrm{s}$ when we consider contribution of ion temperatures only. This is much smaller than the Alfvén velocity, $1030 \mathrm{~km} / \mathrm{s}$.) We show that the phase velocity is larger than the maximum limit which we can determine from magnetic fields measured by the multiple spacecraft. (4) The $E / B$ ratio is the same as the Alfvén velocity when the contribution from the ion acoustic velocity is negligible. As examined above, the $E / B$ ratio is about one-half of the Alfvén speed. (5) The magnetic and electric field variations are perpendicular to each other. This does not hold in our observations because the direction of the Poynting flux is sometimes variable (Figure 7). Although not all of the above five points are always satisfied, we suggest that the most likely explanation is that the waves in our observations are the fast mode waves with multiple propagation directions. It should be noted that, for this event, the existence of a static magnetic structure to reduce the $E / B$ ratio is not supported because the structure we observed 
propagates much faster than the convection velocity. (A mean value of the convection is $11 \mathrm{~km} / \mathrm{s}$.)

[21] We next consider possible generation mechanisms for these compressional waves. These waves are observed when the solar wind speed is $440 \mathrm{~km} / \mathrm{s}$. According to Engebretson et al. [1995], broadband waves are often observed with the solar wind speed above $400 \mathrm{~km} / \mathrm{s}$, which is consistent with our result. However, on one point, the similarity between the waves reported here and those reported by Engebretson et al. [1995] breaks down. Although both waves have broadband spectra, the former consists of oscillations of brief duration, while the latter often continues for a time of the order of hours. Another possible source in the solar wind related to these waves is the variation of dynamic pressure [Farrugia et al., 1989]. In our case, the dynamic pressure changes between 2 and $5 \mathrm{nPa}$ during 2030-2130 UT. The resulting displacements of the magnetopause should cause fast mode waves to be launched into the magnetosphere [Southwood and Kivelson, 1990]. After the waves propagate across the field line into the inner magnetosphere, a part of the waves were reflected because of the gradient of the Alfvén velocity, according to these authors. If the waves propagating in multiple directions coexist, it is possible that the $E / B$ ratio becomes smaller than the Alfvén velocity.

\section{Concluding Remarks}

[22] In this study, we have examined two events of broadband ULF waves around the polar cap boundary. The waves are observed at the frequency $<100 \mathrm{mHz}$. We mainly examined wave characteristics around the period of 5 minutes because analysis with multiple spacecraft data is useful. We have inferred that the wave mode is not the same in the two examples, even though the waveforms and the spacecraft locations are similar. We have restricted the possible modes and generation mechanisms by introducing data from multiple spacecraft and instruments. In the first event on 26 March 2001, the phase velocity calculated from multiple spacecraft data is almost perpendicular to the magnetic field and is much smaller than the $E / B$ ratio. The $E / B$ ratio is $\sim 43 \%$ of the Alfvén velocity. The direction of the Poynting flux is along the magnetic field toward the ionosphere on average, but part of it is away from the ionosphere. The waves are suggested to be Alfvén waves. Kinetic effects are negligible in the region the waves are observed. We propose that the origin of these waves is reconnection. The intermittent observations of these waves indicate that the reconnection was transient. In the second event on 4 March 2001, compressional components exist in addition to transverse components. The phase velocity is too large ( $>\sim 80 \mathrm{~km} / \mathrm{s}$ ) to be determined reliably from magnetic fields recorded at the multiple spacecraft. The $E / B$ ratio is smaller than the Alfven velocity. The direction of the Poynting flux is oblique to the magnetic field. The waves are suggested to be the fast mode waves. Our possible identification of fast mode waves is a new feature in Cluster studies. It is inferred that the source of the waves is solar wind pressure variations.

[23] So far, we have mainly discussed the lowest frequency end of the broadband waves. When we examine frequency dependence of the $E / B$ ratio, this value tends to increase as frequency increases. This point for the first event was in agreement with Chaston et al. [2005], in which frequency dependence of the $E / B$ ratio is interpreted in terms of the kinetic feature of the Alfvén waves. It is natural that the wave characteristics of the broadband waves are variable depending on frequency.

[24] In our study, we chose events in which the multiple spacecraft comparison is possible. An event with relatively stable time lags between spacecraft is chosen for the first event. It is left for future work to analyze events with small spatial/temporal size or with larger frequency/wave number in which observations are not similar between multiple spacecraft. Period of data with small separation between spacecraft is necessary in this case. As an opposite case, the magnetic profile is mostly similar between spacecraft when the wave events have a large spatial/temporal size. This is true for our second event on 4 March 2001. Although we limited the possible range of the phase velocity, it is necessary to use data with larger separation between spacecraft to determine the exact phase velocity. However, this might introduce complications because larger separation causes variation of the background field, which merges into the wave components. Although the phase velocity which is possible to determine is limited, this study shows the increased opportunity for the analysis of broadband waves using data from multiple spacecraft.

[25] Finally, the following future work might be suggested. For shear Alfvén waves, further identification of the wave generation mechanisms is desirable. Observations made simultaneously at the magnetopause and inside the magnetosphere are preferable, together with the relevant simulation works. For the fast mode waves, an open question concerns the way the energy of these waves propagates in the magnetosphere. Since the fast mode waves are known to couple with the shear Alfvén waves, observational signatures of this aspect should be examined and compared with theories in realistic situations.

[26] Acknowledgments. We thank J. M. Quinn and K.-H. Glassmeier for their useful comments on the manuscript to improve its quality. We are grateful to N. F. Ness and D. J. McComas for the magnetic field and plasma data from ACE. These data are obtained from the CDAWeb site. This work was supported by NASA through grants NAG5-9960, NNG04GA46G, and NNG05GG25G

[27] Zuyin Pu thanks the reviewers for their assistance in evaluating this paper.

\section{References}

Anderson, B. J., M. J. Engebretson, S. P. Rounds, L. J. Zanetti, and T. A. Potemra (1990), A statistical study of Pc3-5 pulsations observed by the AMPTE/CCE magnetic field experiment 1. Occurrence distributions, J.Geophys. Res., 95, 10495-10523.

Balogh, A., et al. (2001), The Cluster magnetic field investigation: Overview of in-flight performance and initial results, Ann. Geophys., 19, 6591.

Born, M., and E. Wolf (1999), Principle of Optics, 7th ed., 952 pp., Cambridge University Press, Cambridge.

Bosqued, J. M., et al. (2005), Multipoint observations of transient reconnection signatures in the cusp precipitation: A Cluster-IMAGE detailed case study, J. Geophys. Res., 110, A03219, doi:10.1029/2004JA010621.

Chaston, C. C., J. W. Bonnell, C. W. Carlson, J. P. McFadden, and R. E. Ergun (2003), Properties of small-scale Alfvén waves and accelerated electrons from FAST, J. Geophys. Res., 108(A4), 8003, doi:10.1029/ 2002JA009420

Chaston, C. C., et al. (2005), Energy deposition by Alfvén waves into the dayside auroral oval: Cluster and FAST observations, J. Geophys. Res., 110, A02211, doi:10.1029/2004JA010483. 
Cowley, S. W. H. (2000), Magnetosphere-ionosphere interactions: A tutorial review (2000), in Magnetospheric Current Systems, Geophys. Monograph 118, edited by S. Ohtani, R. Fujii, M. Hesse, and R. L. Lysak, pp. 91-106, American Geophysical Union, Washington.

Décréau, P. M. E., et al. (2001), Early results from the Whisper instrument on Cluster: An overview, Ann. Geophys., 19, 1241-1258.

Dombeck, J., C. Cattell, J. R. Wygant, A. Keiling, and J. Scudder (2005), Alfvén waves and Poynting flux observed simultaneously by Polar and FAST in the plasma sheet boundary layer, J. Geophys. Res., 110, A12S90, doi:10.1029/2005JA011269.

Engebretson, M. J., W. J. Hughes, J. L. Alford, E. Zesta, L. J. Cahill Jr., R. L. Arnoldy, and G. D. Reeves (1995), Magnetometer array for cusp and cleft studies observations of the spatial extent of broadband ULF magnetic pulsations at cusp/cleft latitudes, J. Geophys. Res., 100, $19,371-19,386$

Eriksson, A. I. (1998), Spectral Analysis, in Analysis Methods for MultiSpacecraft Data, ISSI Sci. Rep. SR-001, edited by G. Paschmann and P. W. Daly, pp. 5-42, ESA Publications, Noordwijk, Netherlands.

Eriksson, A. I., et al. (2006), Electric field measurements on Cluster: Comparing the double-probe and electron drift techniques, Ann. Geophys., 24 $275-289$

Farrugia, C. J., M. P. Freeman, S. W. H. Cowley, D. J. Southwood, M. Lockwood, and A. Etemadi (1989), Pressure-driven magnetopause motions and attendant response on the ground, Planet. Space Sci., 37, $589-607$

Gosling, J. T., M. F. Thomsen, S. J. Bame, R. C. Elphic, and C. T. Russell (1990), Plasma flow reversals at the dayside magnetopause and the origin of asymmetric polar cap convection, J. Geophys. Res., 95, 8073-8084.

Hasegawa, A. (1969), Drift mirror instability in the magnetosphere, Phys. Fluids, 12, 2642-2650.

Heyn, M. F., H. K. Biernat, R. P. Rijnbeek, and V. S. Semenov (1988), The structure of reconnection layers, J. Plasma Phys., 40, 235-252.

Kivelson, M. G. (1995), Pulsations and magnetohydrodynamic waves, in Introduction to Space Physics, edited by M. G. Kivelson and C. T. Russell, pp. 330-355, Cambridge University Press, Cambridge.

Lysak, R. L. (1991), Feedback instability of the ionospheric resonant cavity, J. Geophys. Res., 96, 1553-1568.

McComas, D. J., S. J. Bame, P. Baker, W. C. Feldman, J. L. Phillips, P. Riley, and J. W. Griffee (1998), Solar wind electron proton alpha monitor (SWEPAM) for the advanced composition explorer, Space Sci. Rev., 86, 563-612.
Paschmann, G., et al. (2001), The Electron Drift Instrument on Cluster: overview of first results, Ann. Geophys., 19, 1273-1288.

$\mathrm{Pu}, \mathrm{Z}$., and Y. Zhou (1986), The kinetic Alfvén wave instability driven by a sheared plasma flow and the associated anomalous transport, Sci. Sin., Ser. $A, 29,301-311$.

Puhl-Quinn, P. A., H. Matsui, G. Paschmann, M. Bouhram, K. Glassmeier, and E. Georgescu (2004), ULF wave activity in the terrestrial polar cap and polar cap boundary regions as observed by Cluster, Eos Trans. $A G U$, 85(47), Fall Meet. Suppl., Abstract SM33A-1245.

Rème, H., et al. (2001), First multispacecraft ion measurements in and near the Earth's magnetosphere with the identical Cluster ion spectrometry (CIS) experiment, Ann. Geophys., 19, 1303-1354.

Robinson, R. M., and R. R. Vondrak (1984), Measurements of E region ionization and conductivity produced by solar illumination at high latitudes, J. Geophys. Res., 89, 3951-3956.

Smith, C. W., J. L'Heureux, N. F. Ness, M. H. Acuña, L. F. Burlaga, and J. Scheifele (1998), The ACE magnetic fields experiment, Space Sci. Rev., 86, 613-632.

Soucek, J., T. Dudok de Wit, M. Dunlop, and P. Décréau (2004), Local wavelet correlation: application to timing analysis of multi-satellite CLUSTER data, Ann. Geophys., 22, 4185-4196.

Southwood, D. J., and M. G. Kivelson (1990), The magnetohydrodynamic response of the magnetospheric cavity to changes in solar wind pressure, J. Geophys. Res., 95, 2301-2309.

Stasiewicz, K., C. E. Seyler, F. S. Mozer, G. Gustafsson, J. Pickett, and B. Popielawska (2001), Magnetic bubbles and kinetic Alfven waves in the high-latitude magnetopause boundary, J. Geophys. Res., 106, 29,50329,514 .

W. Baumjohann, Space Research Institute, Austrian Academy of Sciences, Schmiedlstr. 6, 8042 Graz, Austria

P. M. E. Décréau, Laboratoire de Physique et Chimie de l'Environnement (LPCE), CNRS and University of Orléans, Orléans, France.

C. J. Farrugia, H. Matsui, C. G. Mouikis, P. A. Puhl-Quinn, and R. B. Torbert, Space Science Center, University of New Hampshire, Durham, NH 03824, USA. (hiroshi.matsui@unh.edu)

E. A. Lucek, Imperial College, London, UK.

G. Paschmann, Max-Planck-Institut für extraterrestrische Physik, Garching, Germany. 